Mappemonde

Revue trimestrielle sur l'image géographique et les formes du territoire

$126 \mid 2019$

Varia

\title{
Tordesillas, la bourgade où Castillans et Portugais se sont partagé l'Amérique
}

Hervé Théry

\section{OpenEdition}

Journals

Édition électronique

URL : https://journals.openedition.org/mappemonde/1197

DOI : 10.4000/mappemonde.1197

ISSN : $1769-7298$

Éditeur

UMR ESPACE

\section{Référence électronique}

Hervé Théry, «Tordesillas, la bourgade où Castillans et Portugais se sont partagé l'Amérique », Mappemonde [En ligne], 126 | 2019, mis en ligne le 06 novembre 2019, consulté le 28 juin 2022. URL : http://journals.openedition.org/mappemonde/1197; DOI : https://doi.org/10.4000/mappemonde. 1197

Ce document a été généré automatiquement le 29 septembre 2020.

\section{cc) (1)}

La revue Mappemonde est mise à disposition selon les termes de la Licence Creative Commons Attribution - Pas d'Utilisation Commerciale - Partage dans les Mêmes Conditions 4.0 International. 


\title{
Tordesillas, la bourgade où Castillans et Portugais se sont partagé l'Amérique
}

\author{
Hervé Théry
}

1 Pour qui y arrive aujourd'hui, Tordesillas, petite ville de Castille située à un peu moins de $200 \mathrm{~km}$ au nord-ouest de Madrid, entre Valladolid, Salamanque et Ségovie (figure 2), ressemble à n'importe quelle autre petite cité des bords du fleuve Douro, dans la province espagnole de Valladolid, avec un centre historique groupé autour de la Plaza Mayor (place centrale) et des périphéries modernes sans grand intérêt. Mais son nom est très connu de qui s'intéresse à l'histoire de l'Amérique du Sud, car c'est là qu'en 1494 le Royaume de Castille et le Portugal ont signé le «Traité de Tordesillas » par lequel ils se sont mis d'accord pour ce partage les territoires qu'ils avaient découverts ou allaient découvrir au Nouveau Monde. Et s'y rendre aujourd'hui permet de chercher la trace de cet évènement dans le paysage de 2018, cinq siècles (et quart) plus tard, et de constater qu'il y est bien vivant.

Figure 1. Tordesillas, vue générale depuis les rives du Douro

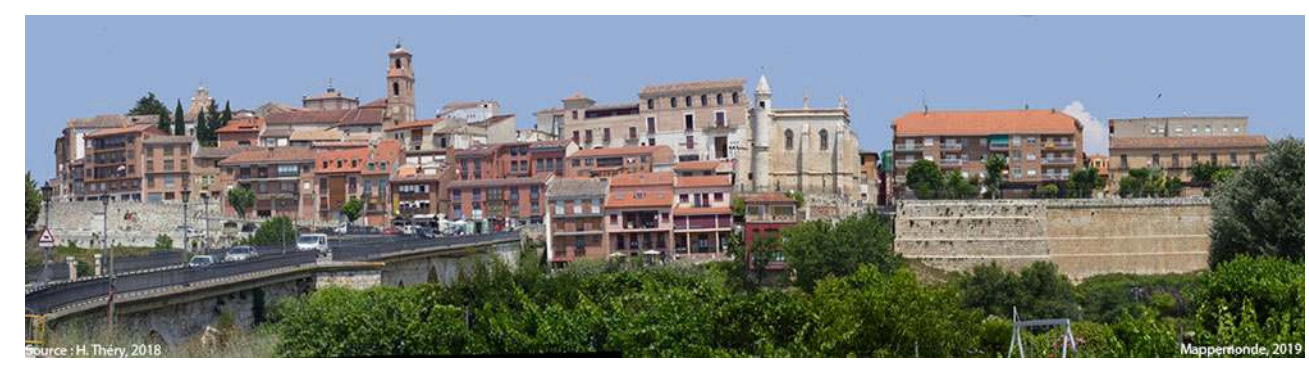

Au centre, de part et d'autre du clocher blanc, les Casas del Tratado, où a été signé le Traité de Tordesillas.

Hervé Théry, 2018 


\section{Pourquoi Tordesillas?}

2 Si l'on peut s'étonner du choix d'une modeste bourgade pour la signature d'un document de si grande portée ${ }^{1}$, il n'était toutefois pas fortuit, car la situation de la ville en faisait un endroit idéal pour les négociations entre les deux royaumes. Comme l'indique Margarita Gokun (2017), citant Miguel Angel Zalama, directeur du Centre Tordesillas pour les relations ibéro-américaines «Tordesillas était au carrefour de routes très importantes de l'époque [...] Il y avait déjà là un palais où tout indique que le traité a été signé ». Celui-ci est maintenant connu comme Casas del Tratado (voir cidessous), mais la présence d'un endroit confortable et la facilité d'accès peuvent ne pas avoir été les seules raisons pour lesquelles les monarques espagnols ont choisi

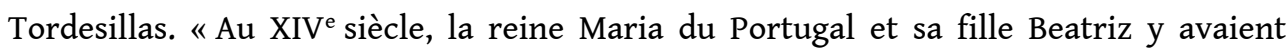
habité, la ville avait donc un lien historique avec le pays et il est possible que la Castille ait fait un geste politique pour que les Portugais se sentent plus à l'aise ", comme l'explique à Margarita Gokun Ricardo Piqueras Céspedes, historien de l'Université de Barcelone.

3 Tordesillas est en effet située dans une région riche d'histoire: Valladolid a été la résidence des rois de Castille depuis le XVe siècle jusqu'à ce que Philippe II fasse de Madrid la capitale du royaume, en 1561, et c'est à Ségovie qu'Isabelle la Catholique a été proclamée reine de Castille le 13 décembre 1474. À Medina del Campo se trouve le château de La Mota où Isabelle de Castille (connue sous le nom d'Isabel la Católica) a passé ses derniers jours. L'Université de Salamanque, fondée par Alphonse IX de León en 1218, est une des plus anciennes en Europe (après Bologne, Paris, Oxford et Cambridge), et la première à avoir obtenu le titre d'Université. Avila, toute proche a vu naître Teresa de Cepeda y Ahumada (plus connue sous le nom de sainte Thérèse d'Avila).

4 Valladolid est par ailleurs liée à la conquête ouverte par le traité de Tordesillas puisque c'est là qu'a eu lieu la « controverse de Valladolid » qui porta en 1550 sur le statut des Indiens d'Amérique : avaient-ils une âme, et si oui, pouvait-on tout de même les réduire en esclavage? Elle opposa Bartolomé de Las Casas, qui défendait des Indiens, au dominicain et théologien Juan Ginés de Sepúlveda. Bien que sans portée réelle à l'époque elle a eu une belle postérité dans l'histoire des idées. 
Figure 2. Situation de Tordesillas

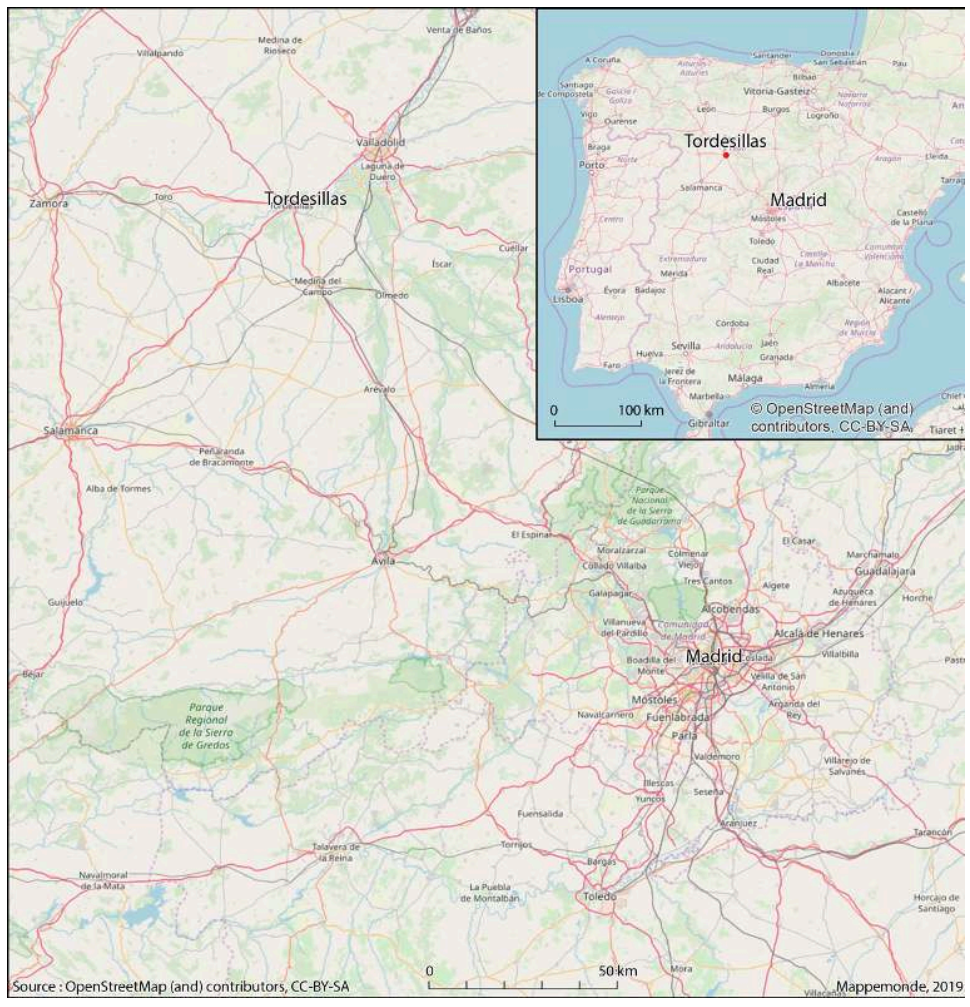

Source : Google maps

5 La ville elle-même avait déjà une belle histoire derrière elle avant le Traité, elle apparaît dans les documents du haut Moyen Âge sous le nom d'Oterium de Sellis, un toponyme désignant un rocher fortifié permettant de surveiller les terres environnantes. Et elle s'est illustrée vers 939 lorsque, lors de la Reconquista (menée contre les Maures qui occupaient alors l'Espagne), Abderramán III y perdit une bataille contre les Rois de León et de Navarre. Cette victoire des rois chrétiens fit passer les territoires de Tordesillas et de Zamora sous leur domination, longtemps disputée et qui ne fut définitive qu'en 995.

\section{Le Traité de Tordesillas}

Le traité de Tordesillas, négocié sous l'égide de la papauté et signé le 7 juin 1494, partageait le Nouveau Monde entre la Castille et le Portugal: ces terres inconnues étaient considérées comme terra nullius, sans tenir compte des droits des habitants qui y avaient été "découverts ». L'accord définit comme ligne de partage un méridien passant à 370 lieues (environ 1800 kilomètres) à l'ouest des îles du Cap-Vert, méridien qui se situerait aujourd'hui à $46^{\circ} 37^{\prime}$ de longitude ouest. Ce faisant, le traité plaçait le futur Brésil, découvert peu de temps après ${ }^{2}$ par le Portugais Pedro Alvares Cabral, sous souveraineté portugaise.

7 Ce traité avait dû être négocié pour résoudre les conflits nés de la toute récente découverte du Nouveau Monde par Christophe Colomb, en 1492. Depuis la prise de Ceuta, au Maroc, en 1415, le Portugal avait pris de l'avance sur sa rivale dans les expéditions maritimes vers le Sud, découvert les Açores au début du XVe siècle, exploré 
méthodiquement la côte africaine et était en passe de contourner le Cap de Bonne Espérance ${ }^{3}$. Dès 1481, la bulle pontificale Æterni regis avait garanti au Portugal toutes les terres découvertes au sud des îles Canaries.

Mais le voyage de Christophe Colomb changea la donne et le pape Alexandre VI, d'origine espagnole, en prit acte sans tarder. Dès le 4 mai 1493, deux semaines à peine après le retour du Gênois, il annula la bulle Aeterna regis et la remplaça par la bulle Inter Caetera selon laquelle les nouvelles terres découvertes situées à l'ouest d'un méridien passant à 100 lieues des îles du Cap-Vert reviendraient à la Castille, celles situées à l'est restant acquises au Portugal. Cette répartition avait mécontenté le roi Jean II de Portugal, qui entama alors des négociations avec les Rois catholiques. Il faisait valoir, afin de le déplacer vers l'ouest, que ce méridien (ou plus exactement son équivalent de l'autre côté du globe, ou "antiméridien») restreignait les prétentions espagnoles en Asie, notamment sur l'archipel des Moluques, riche en épices. Il obtint ainsi que la ligne soit reportée à 370 lieues à l'ouest du Cap-Vert, ce qui augmentait sensiblement la part du Portugal en Amérique du Sud, comme cela apparut plus tard ${ }^{4}$.

Figure 3. Lignes de partage

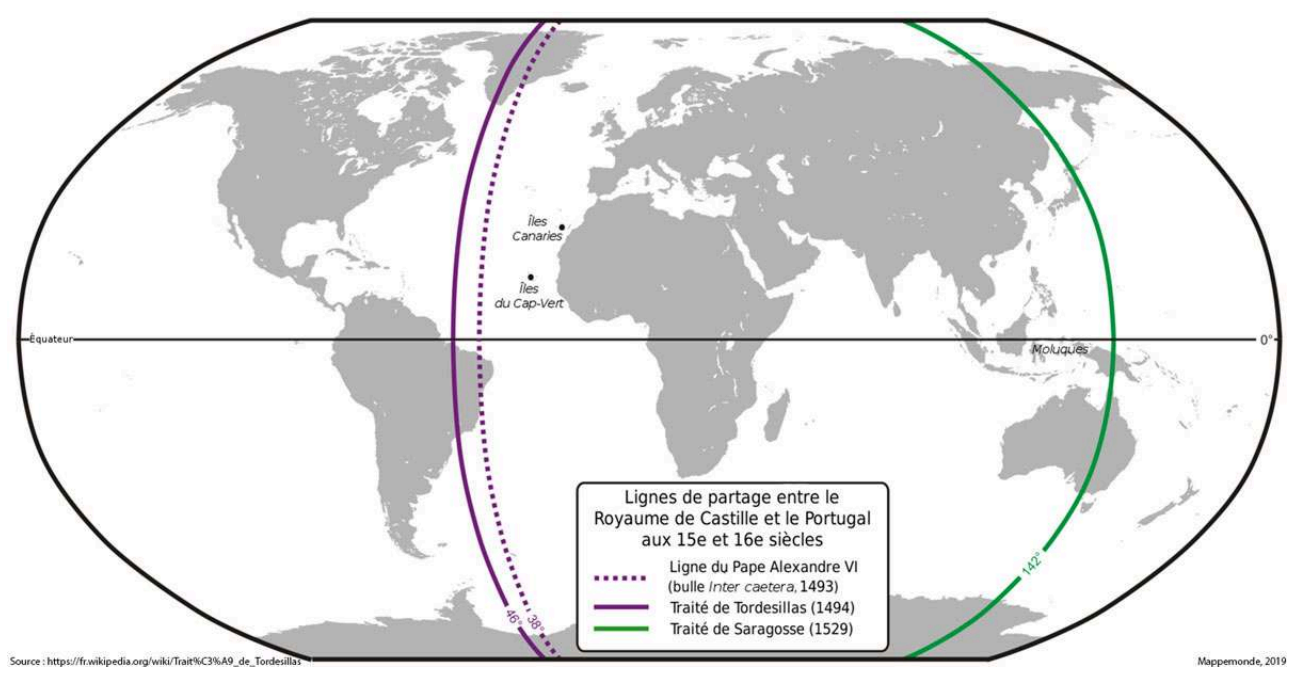

Source : https://fr.wikipedia.org/wiki/Trait\%C3\%A9_de_Tordesillas

9 Le même jour que « le » traité portant sur le partage de l'Atlantique les deux puissances en ont signé un autre, toujours à Tordesillas, qui résolvait les litiges entre les deux royaumes au sujet de leurs intérêts africains : ils se partageaient d'avance le royaume de Fez (Maroc) en vue de leurs futures conquêtes et réglaient les droits de la pêche et de la navigation sur la côte atlantique. Ce pacte africain avait une grande valeur pour la Castille puisque, comme l'indique le site de l'Ayuntamiento de Tordesillas, « il y avait alors à peine deux ans que les Rois Catholiques avaient conclu la Reconquista par l'annexion de Grenade et cet accord avec le Portugal délimitait la zone de la future conquête et de l'expansion du christianisme hispanique face à l'Islam en Afrique du Nord, objectif prioritaire de la monarchie espagnole.

10 L'acte final des négociations a été conclu sans la présence des rois, qui avaient délégué leurs pouvoirs à des représentants plénipotentiaires. [...] Bien que les accords signés le 7 juin aient été fermes, puisque les délégués avaient les pleins pouvoirs, les deux côtés avaient décidé de se donner un délai raisonnable pour cela : 50 jours pour le traité africain et 100 jours pour le traité océanique, puisqu'il était nécessaire d'attendre de 
savoir ce que les bateaux castillans qui naviguaient alors sur l'Océan Atlantique avaient découvert jusqu'au 20 juin ». Celui-ci a donc été ratifié (à Arevalo, à $60 \mathrm{~km}$ de Tordesillas) par le roi Ferdinand II d'Aragon et la reine Isabelle de Castille le 2 juillet 1494, puis dans sa version portugaise, à Setúbal, par le roi Jean II de Portugal, le 5 septembre 1494 et finalement approuvé, le 24 janvier 1506, par le Pape Jules II dans la nouvelle bulle Ea quae pro bono pacis. Les originaux du traité sont conservés aux archives de Torre do Tombo (à Lisbonne) et aux Archives générales des Indes (à Séville).

Figure 4. Le traité de Tordesillas
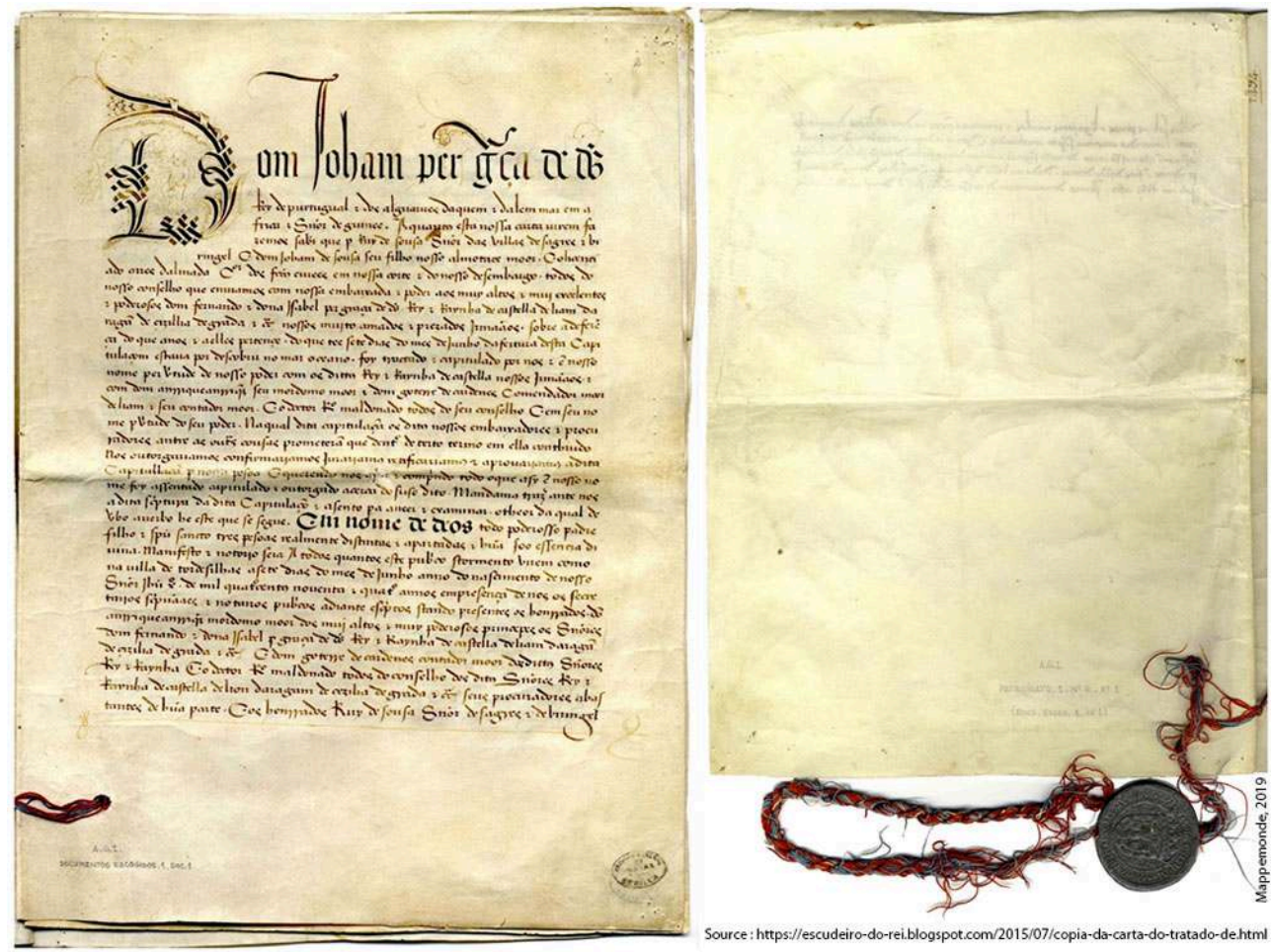

Première et dernière page (avec le Sceau royal) de la version portugaise du Traité.

Source : https://escudeiro-do-rei.blogspot.com/2015/07/copia-da-carta-do-tratado-de.html

11 Les autres puissances maritimes européennes se voyaient ainsi refuser tout droit sur ces nouvelles terres : François $I^{\text {er }}$ s'en irrita et il déclara «le soleil luit pour moi comme pour les autres. Je voudrais bien voir la clause du testament d'Adam qui m'exclut du partage du monde». Il obtint plus tard du pape Clément VII une interprétation assouplie de la bulle : le traité de Tordesillas ne concernait que «les terres connues et non les terres ultérieurement découvertes par les autres Couronnes ». Et comme, pour François I ${ }^{\text {er }}$, seuls «les lieux habités et défendus» pouvaient être réclamés légitimement, il pouvait appuyer les expéditions des marins bretons et normands en Amérique du Nord et même au Brésil ${ }^{5}$.

Aucun autre État ne reconnut le traité de Tordesillas, mais la suprématie navale de l'Espagne et du Portugal leur permit de le faire respecter pendant un siècle. Il devint toutefois caduc lorsque les autres puissances coloniales ayant des ambitions en Amérique (France, Pays-Bas et Royaume-Uni) se furent dotées de flottes navales assez puissantes pour braver l'interdit des Ibériques et, outre des tentatives généralement frustrées en Amérique du Sud ${ }^{6}$ continuèrent la colonisation en occupant des terres plus 
au nord, aux Antilles et aux actuels États-Unis et du Canada, peu ou pas colonisés par les Espagnols et les Portugais.

\section{Tordesillas aujourd'hui, le souvenir du Traité}

Que reste-t-il aujourd'hui de ce traité dans la ville Tordesillas, dans son paysage, dans son patrimoine matériel et immatériel ? Son souvenir est-il cultivé par les habitants et les autorités actuelles? En ont-ils/elles fait un atout touristique, pour que la principale raison de la notoriété de la bourgade y attire des visiteurs?

Figure 5. Sur la Plaza Mayor, le souvenir du Traité

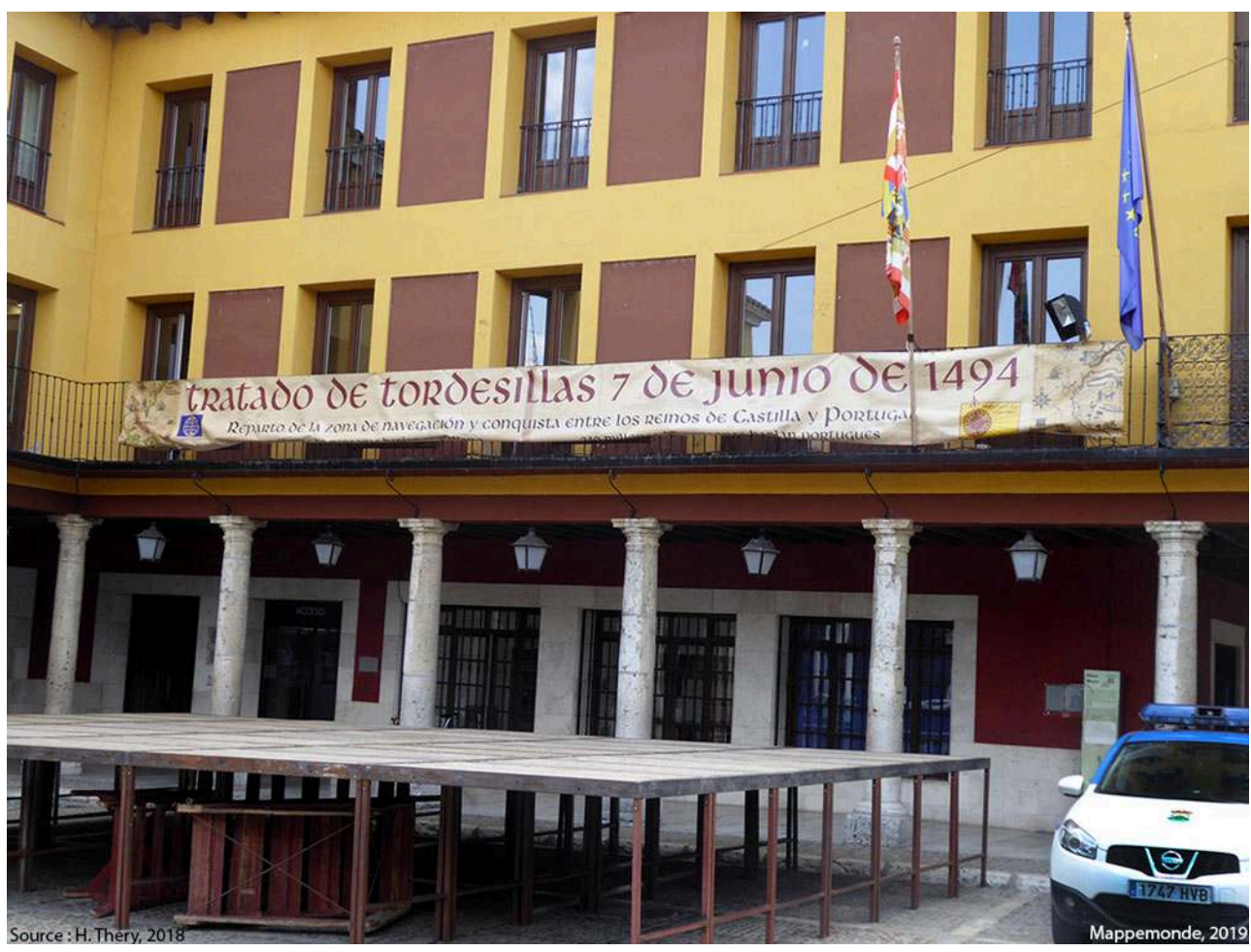

Hervé Théry, 2018

C'est bien le cas, comme on peut s'en rendre compte dès l'arrivée sur la Plaza Mayor, ou une banderole suspendue au-dessus des colonnades qui ceinturent la place, à la hauteur du premier étage, rappelle en grosses lettres la signature du Traité le 7 juin 1494 (figure 5).

Dans les rues de la ville, des panneaux installés par le bureau du tourisme jalonnent le chemin vers le musée et les Casas del Tratado, et devant celles-ci un panneau explicatif relate ce qui s'y est passé, en espagnol, anglais et braille. Qui veut en savoir plus peut, en prenant en photo sur son smartphone du code $Q R$ figurant près de son titre, accéder à une visite virtuelle du musée (figure 6). 
Figure 6. Signalisation des Casas del Tratado
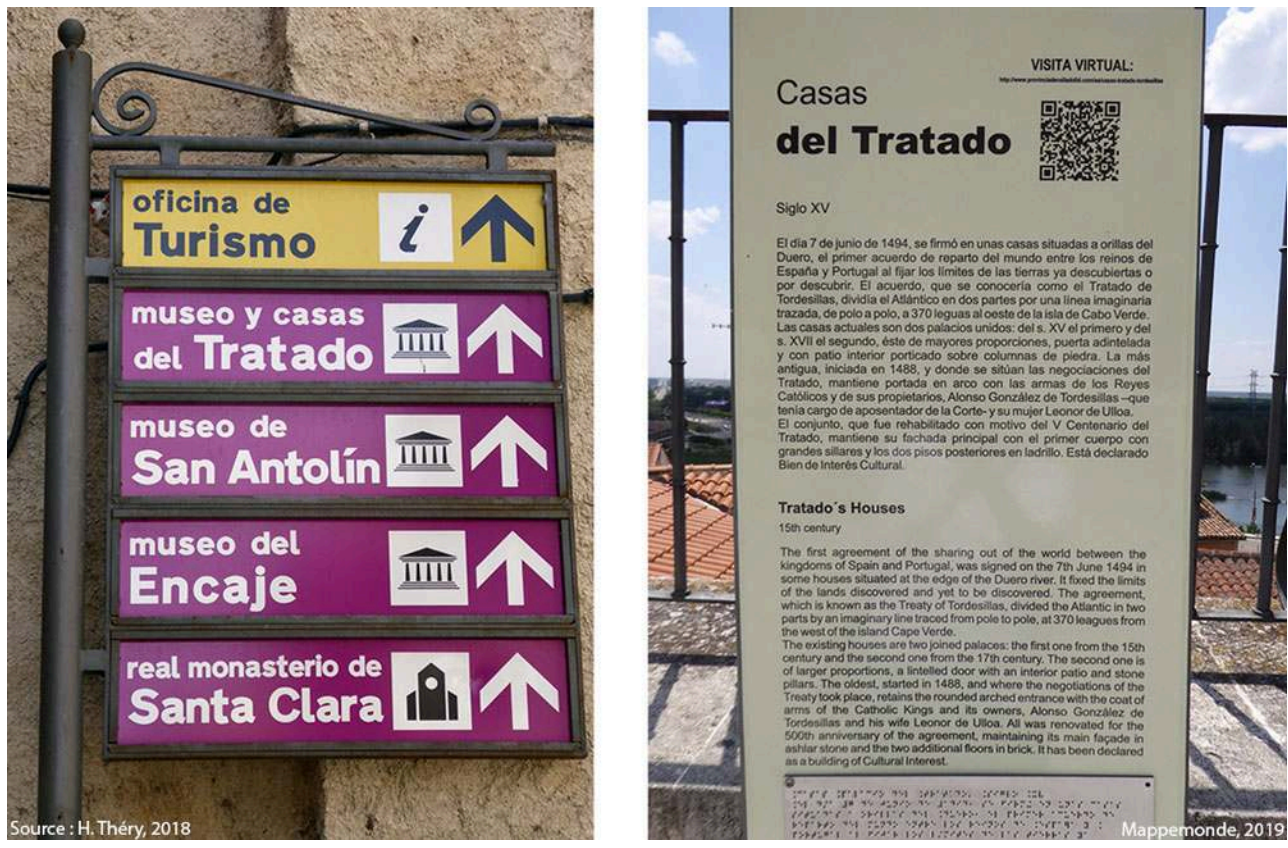

Hervé Théry, 2018

Les Casas del Tratado, où a été (très probablement) signé le Traité, ont été superbement restaurées et mises en valeur, et sont bordées d'une large promenade. Elles donnent sur le fleuve Douro, qui coule en contrebas, et sont bien visibles dès que l'on approche de la ville par son entrée principale (figure 1). Devant la façade du bâtiment principal a été installée une plaque de bronze commémorant la signature du traité (détail agrandi sur la figure 7). 
Figure 7. En façade des Casas del Tratado, la plaque commémorant le Traité

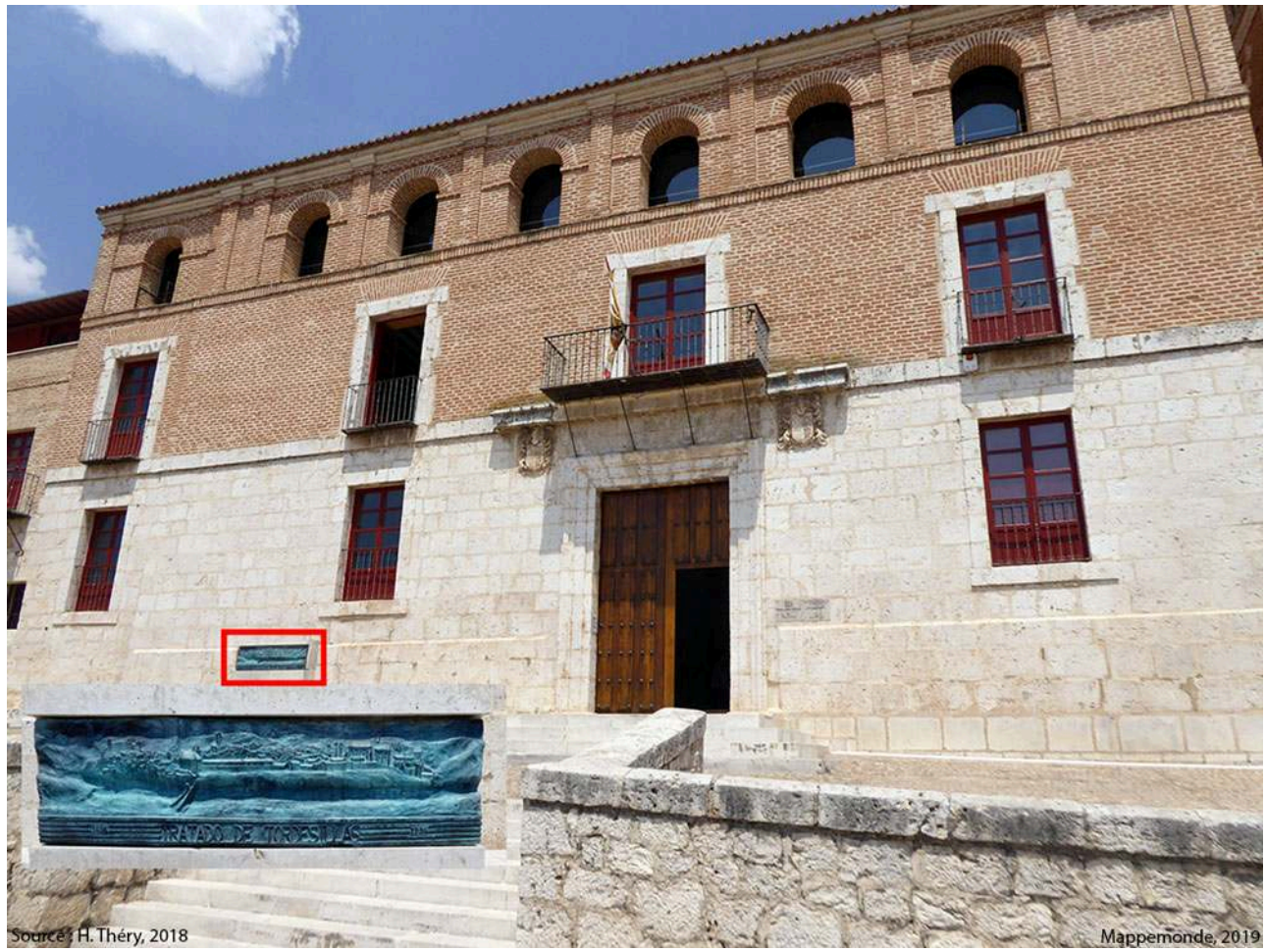

Hervé Théry, 2018

Dans le musée qui occupe le rez-de-chaussée, le cinquième centenaire de la signature du Traité, en 1994, est mentionné par une plaque de bronze et une photographie rappelle qu'il avait été marqué par une double et prestigieuse visite : du côté espagnol, celle du roi Juan Carlos et de son épouse Sofia, du côté portugais celle du Président de la République Mario Soares (figure 8).

Figure 8. Plaque et photo commémorant le $5^{\mathrm{e}}$ Centenaire du Traité
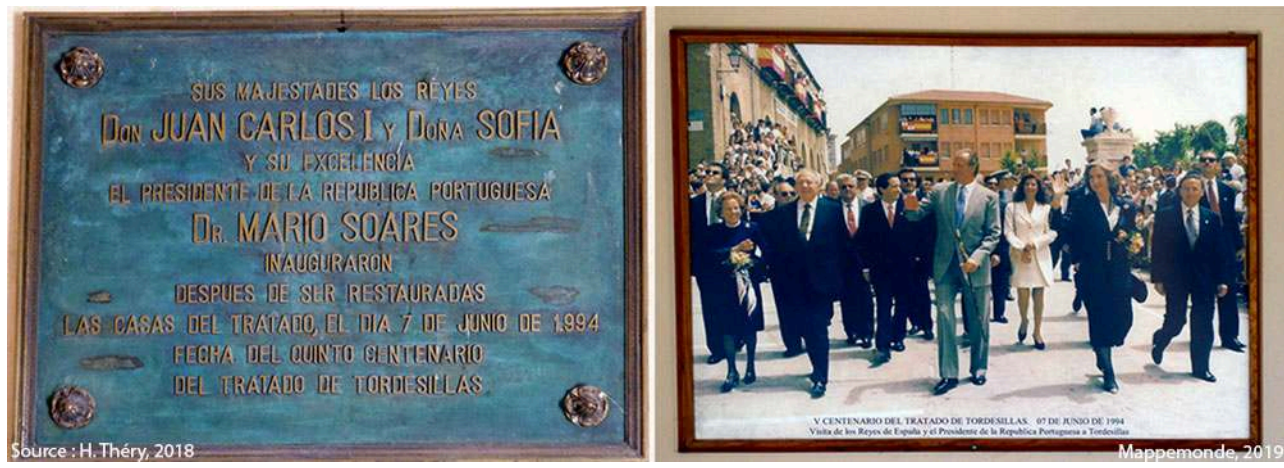

Hervé Théry, 2018

18 Les étages supérieurs du bâtiment (la partie qui n'est pas affectée musée du Traité ou à un musée de maquettes et monuments historiques de la Castille) sont occupés par une bibliothèque où la géographie figure en bonne place... (figure 9) 
Figure 9. La bibliothèque installée dans les Casas del Tratado
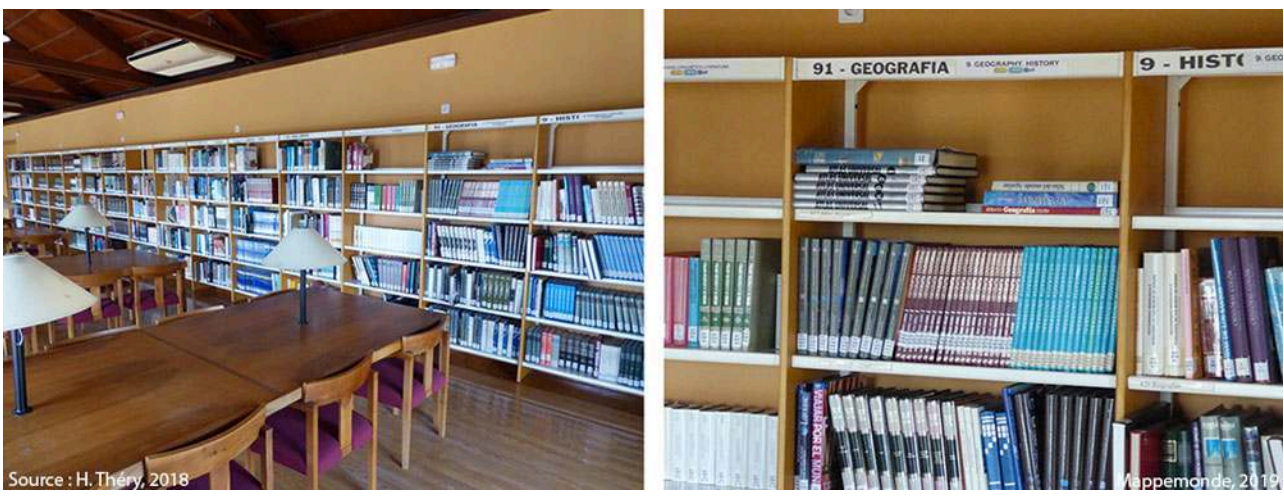

Hervé Théry, 2018

Le souvenir du Traité n'est toutefois pas confiné aux seuls bâtiments où il a été signé, il est aussi évoqué par une série de fresques s'étendant sur plusieurs panneaux qui ornent la rue reliant l'entrée principale de la ville à la Plaza Mayor. Comme celle-ci est parcourue par qui entre dans la ville, la fresque est donc située dans un lieu stratégiquement placé, et forcément vue par tous les visiteurs

Sa première partie, qui mentionne explicitement le traité, sert d'introduction à l'ensemble, elle comporte une rose des vents, un ensemble de lignes évoquant l'Équateur et les Tropiques et surtout un personnage traçant une ligne rouge correspondante au méridien de Tordesillas (figure 11). Un arc formé d'épis de maïs et de tomates rappelle que l'Europe doit ces plantes au Nouveau Monde, d'où les navigateurs du XVI ${ }^{\mathrm{e}}$ siècle les ont rapportées ${ }^{7}$. 
Figure 10. Fresque évoquant le Traité, partie 1

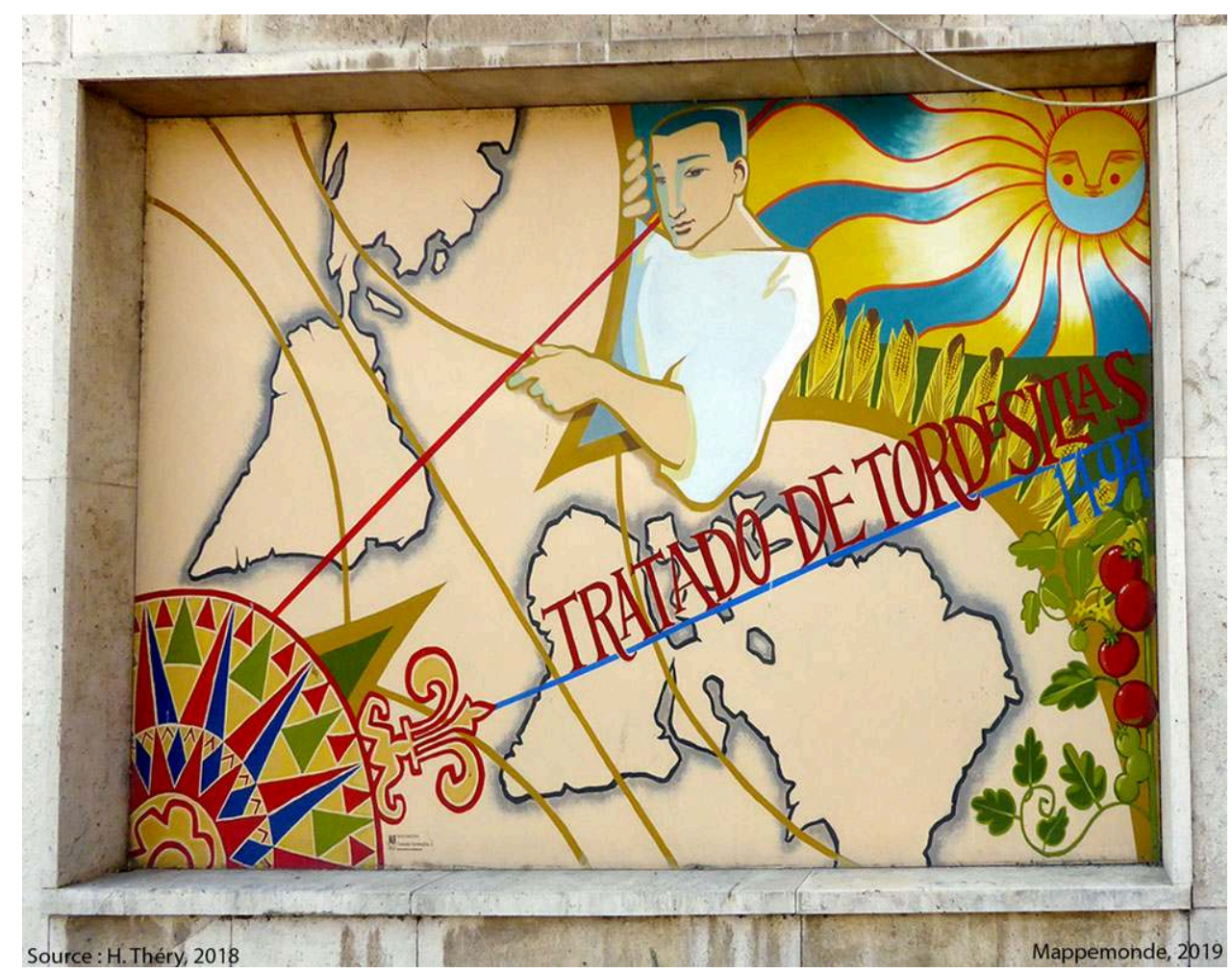

Hervé Théry, 2018

21 La deuxième partie est composée de deux panneaux, séparés par une colonne qui a été astucieusement utilisée pour représenter le méridien : d'un côté des raisins, une croix et un épi de blé se réfèrent à l'Europe, de l'autre des plantes et fruits tropicaux (maïs, tomate et tabac) évoquent l'Amérique du Sud, cette fois accompagnés d'une poterie ornée de motifs indiens. 
Figure 11. Fresque évoquant le Traité, partie 2

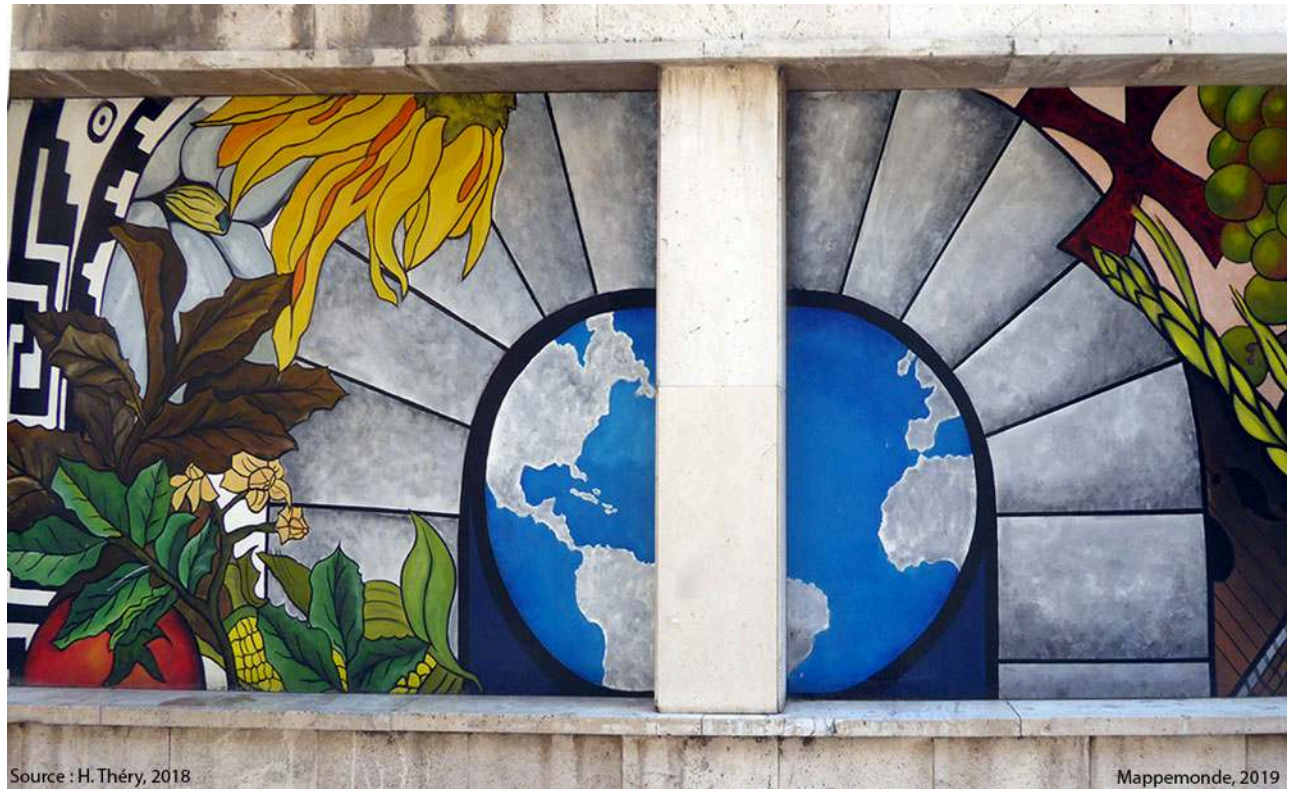

Hervé Théry, 2018

Sur la troisième partie apparaissent les Rois Catholiques espagnols, le roi du Portugal et les trois navires de Christophe Colomb, une autre rose des vents et l'un de ces dragons qui ornaient fréquemment les cartes et portulans de l'époque pour signifier les dangers de ces mers inconnues. Aux deux extrémités, des cartes de la côte du Brésil, d'un côté, et de la péninsule Ibérique, de l'autre, sur laquelle figure discrètement le nom de Tordesillas (agrandi sur le détail la figure 12).

Figure 12. Fresque évoquant le Traité, partie 3

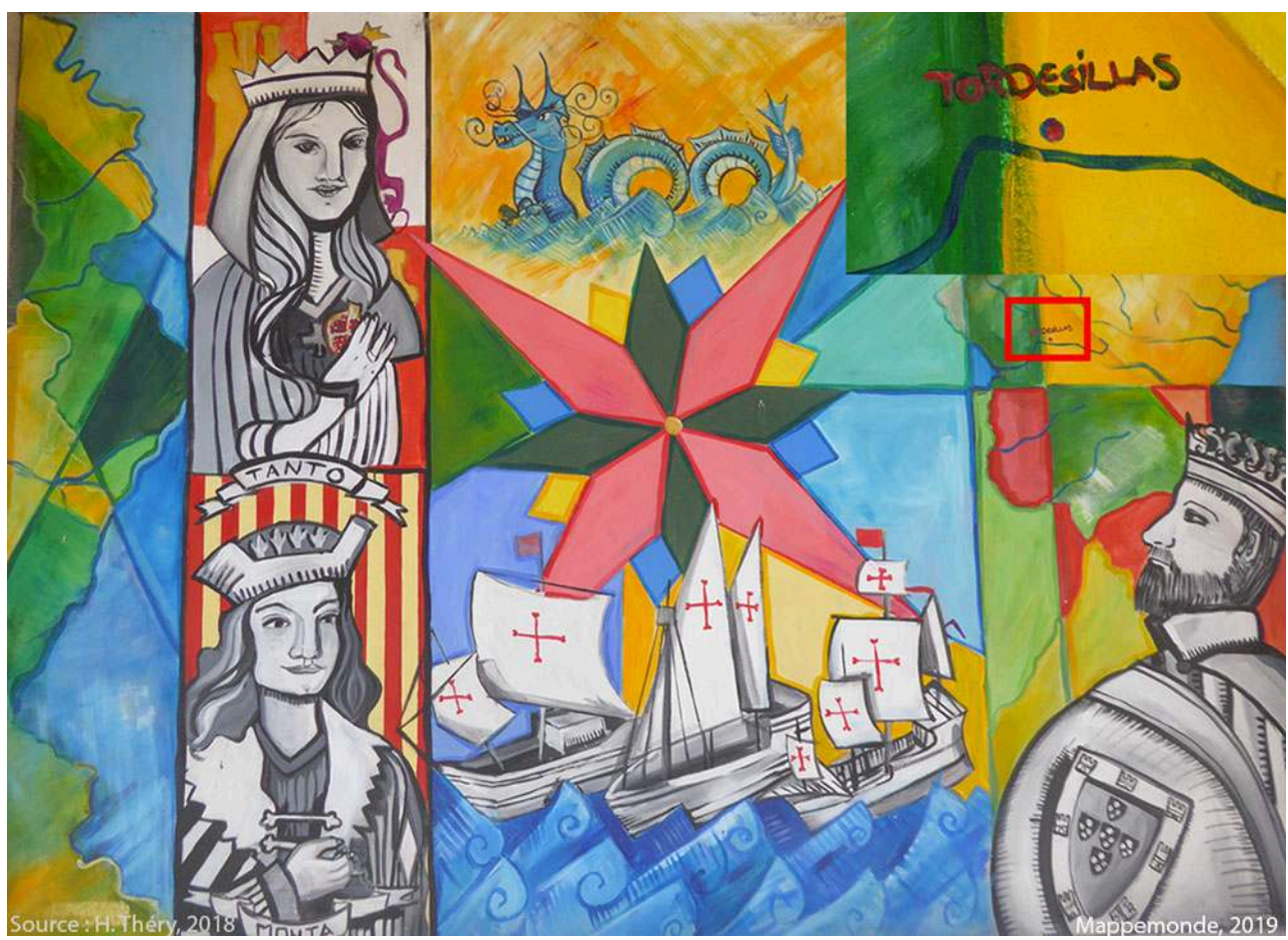

Hervé Théry, 2018 
Finalement, la quatrième partie de la fresque, la plus soignée, est (à mon sens) la plus réussie et la plus touchante. Elle représente le partage du territoire de l'Amérique du Sud sous la forme d'un arrachement, dont le point de départ est une croix plantée dans le sol et d'où partent des fissures que Castillans et Portugais élargissent de force. On passe donc de la célébration de la découverte et de la conquête de nouvelles terres (et plantes) à l'effet produit sur leurs habitants, jusque-là bien peu évoqués. Sans doute est-ce une conséquence d'un changement de perspective qui a pris force lors du cinquième centenaire du voyage de Christophe Colomb, en 1992, quand des organisations indigènes des deux Amériques et leurs sympathisants de part et d'autre de l'Atlantique ont rappelé que le continent "découvert» par les navigateurs européens était déjà occupé depuis plusieurs millénaires. Et donc que le partage du Nouveau Monde entre les puissances ibériques, conclu à Tordesillas deux ans plus tard, avait été vécu par eux de façon moins positive - pour dire le moins - que par les signataires du Traité.

Le nouveau continent est donc ici représenté par une forme triangulaire, dont la base est une montagne couverte de forêts, dont le haut est un visage féminin aux yeux clos, mais dont coulent des larmes qui forment deux rivières, allant respectivement vers l'est vers l'ouest, vers l'Atlantique et le Pacifique. Loin de la glorification du partage du continent entre les deux peuples ibériques, la fresque évoque donc ici avec force la violence faite aux victimes de la " découverte ", représentées par la figure de la déesse Terre, la Pachamama des mythologies andines.

Figure 13. Fresque évoquant le Traité, partie 4

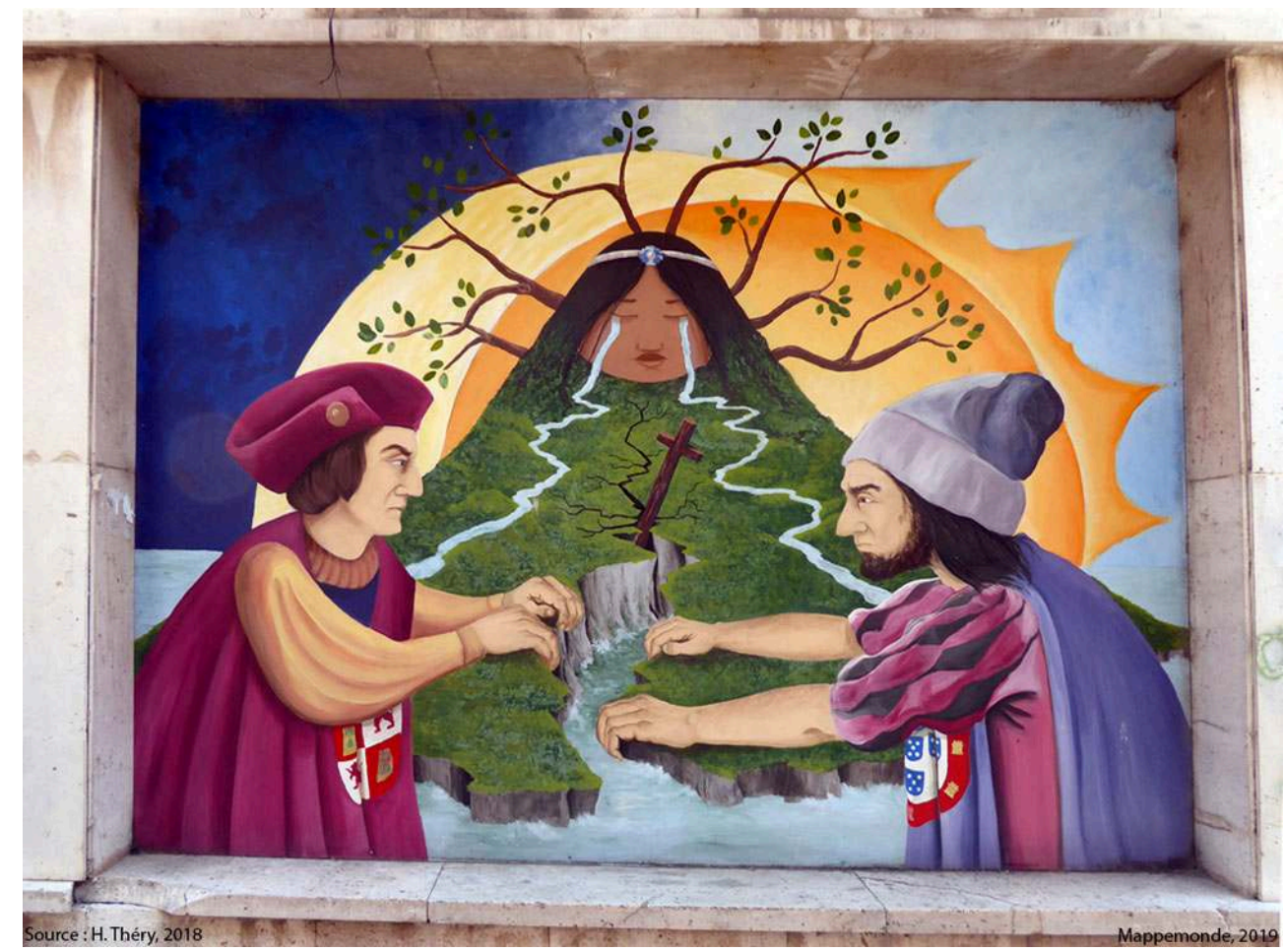

Hervé Théry, 2018 


\section{Tordesillas après le Traité}

La signature de l'un des traités les plus importants de l'histoire n'a pourtant pas fait de Tordesillas, malgré tous les efforts des autorités locales, une destination célèbre et un grand centre touristique. La ville compte un peu plus de 8000 habitants $^{8}$ et elle est surtout citée dans les médias en raison des protestations des groupes de défense des droits des animaux contre le "Toro de la Vega", un festival annuel dans lequel un taureau est traqué dans les rues par des centaines de personnes. Jusqu'en 2016, l'animal était mis à mort à coup de lance, mais cette pratique a été interdite par le gouvernement régional. Sur la place centrale, la banderole défendant cette tradition (au centre de la figure 15) occupe d'ailleurs plus de place que celle qui commémore le Traité (figure 5 et gauche de la figure 14).

Figure 14. Les banderoles de la Plaza Mayor

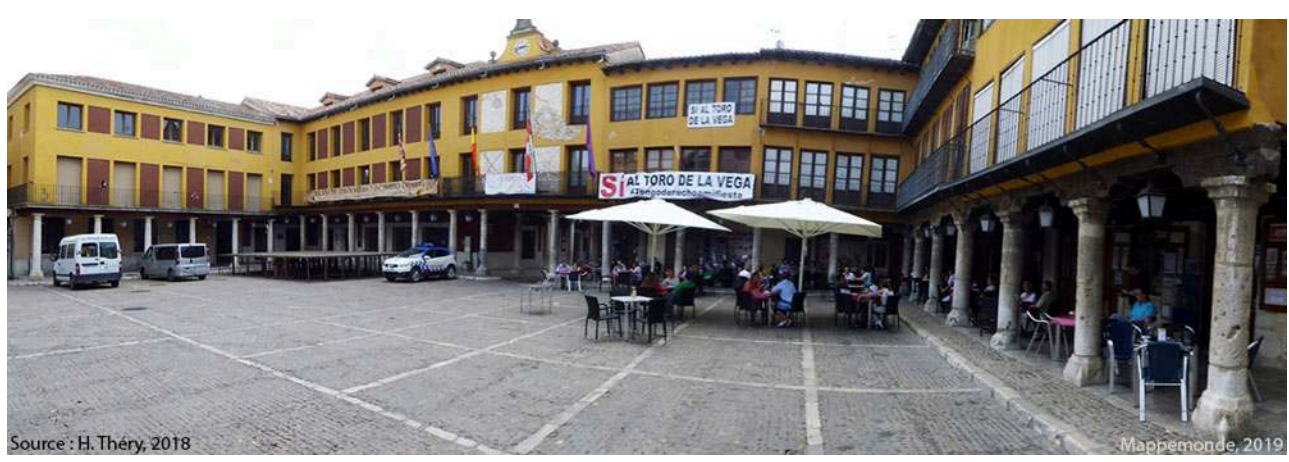

Hervé Théry, 2018

Par ailleurs l'histoire de Tordesillas n'est pas seulement liée au Traité, elle comporte aussi deux épisodes ultérieurs, l'un et l'autre tristes et donc moins susceptibles d'être utilisés pour l'image et la fréquentation de la ville. Un de ses plus beaux bâtiments, le couvent de Santa Clara, a été pendant presque cinquante ans la prison de Joana $1^{\mathrm{e}}$, fille des Rois Catholiques, qui monta sur le trône avec la mort de sa mère en 1504. Déclarée folle, elle y fut cloîtrée en 1509 sur l'ordre de son propre père, et y resta jusqu'à sa mort en 1555, même après que son fils, Charles 1er, fut devenu roi en 1519.

Un autre triste évènement est lié au mauvais souvenir laissé par l'invasion et l'occupation française de 1812, lors des guerres napoléoniennes. Une plaque de bronze (figure 16) est consacrée à un hommage aux habitants de la ville et à l'armée espagnole venue de Galice pour leur victoire contre les troupes françaises d'occupation, dont la capitulation a été reçue dans l'église qui porte la plaque. L'hommage aux habitants est justifié puisque les troupes françaises ont été en grande partie vaincues par la résistance de la population, si vive que c'est alors que le mot espagnol guérilla a été incorporé à la langue française. La plaque indique en outre qu'en cette année $1812^{9}$ la ville a subi l'explosion d'un ermitage et de l'une des arches du pont médiéval sur le Douro, témoignant des dégâts provoqués par cette occupation. 
Figure 15. Les (mauvais) souvenirs de l'occupation française

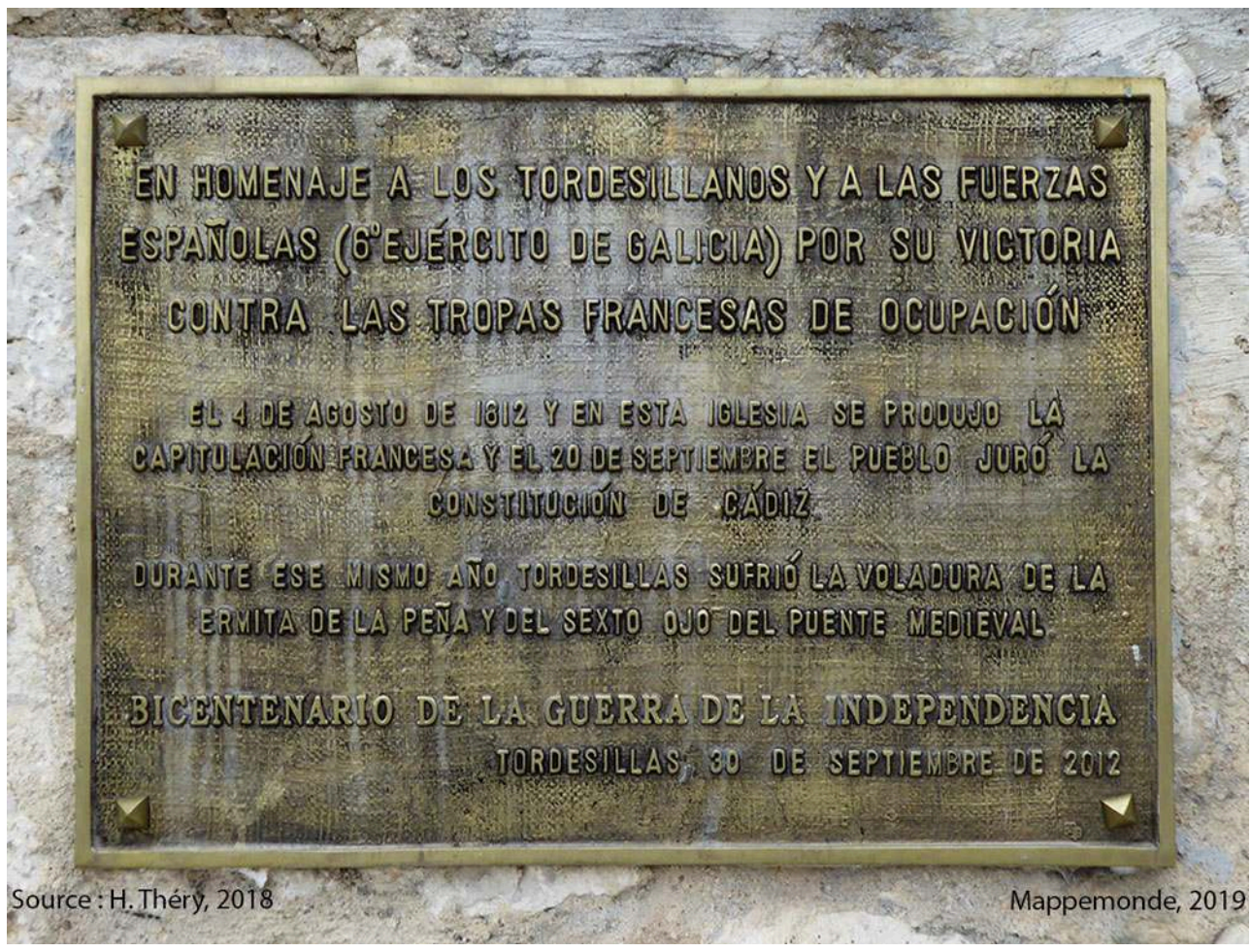

Hervé Théry, 2018

Pour ne pas rester sur cette mauvaise impression, on terminera cette visite à la recherche de la postérité du Traité, le grand évènement de l'histoire de Tordesillas, par une autre image, elle aussi située sur le parcours entre l'entrée de la ville et la Plaza Mayor, un peu avant les fresques mentionnées plus haut.

Elle représente un jeune indien d'Amazonie, portant une coiffure de plumes et le visage décoré de peintures rituelles, encadré par une inscription répétant, à droite et à gauche de son visage, en portugais et en espagnol, les mêmes mots : « bienvenus », « vous » (se combinant sans doute en «bienvenue à vous ») et «Dieu», ce dernier mot évoquant probablement l'évangélisation du continent par les missionnaires catholiques. Un trait vertical au milieu de l'image (et du visage du jeune garçon) représente certainement le méridien, séparant le côté lusophone et le côté hispanophone, une interprétation renforcée par la présence du mot « hablar » (parler), du côté gauche.

Nous n'avons pas pu déterminer ni l'origine ni le sens exact de cette image, on peut choisir de l'interpréter comme une autre représentation du traumatisme de la conquête, qui a séparé les peuples autochtones en deux moitiés parlant chacune la langue de ses conquérants. On peut toutefois choisir de privilégier la bienveillance contenue dans le mot «bienvenue » qui insiste sur l'accueil que ces «bons sauvages », pour reprendre l'expression de Montaigne, ont réservé aux étranges visiteurs venus de l'autre côté de la mer, sans se douter des épreuves qui les attendaient.

1 En tout état de cause, cette image d'un «Indien» (pour reprendre la désignation erronée qui perdure depuis que Christophe Colomb a cru aborder en Inde) rappelle opportunément, au cœur de la ville, qu'y a été prise une décision dont les conséquences ont affecté le destin de peuples vivant de l'autre côté de la planète, et se font encore sentir plus de cinq siècles plus tard. 
Figure . Un Indien à Tordesillas

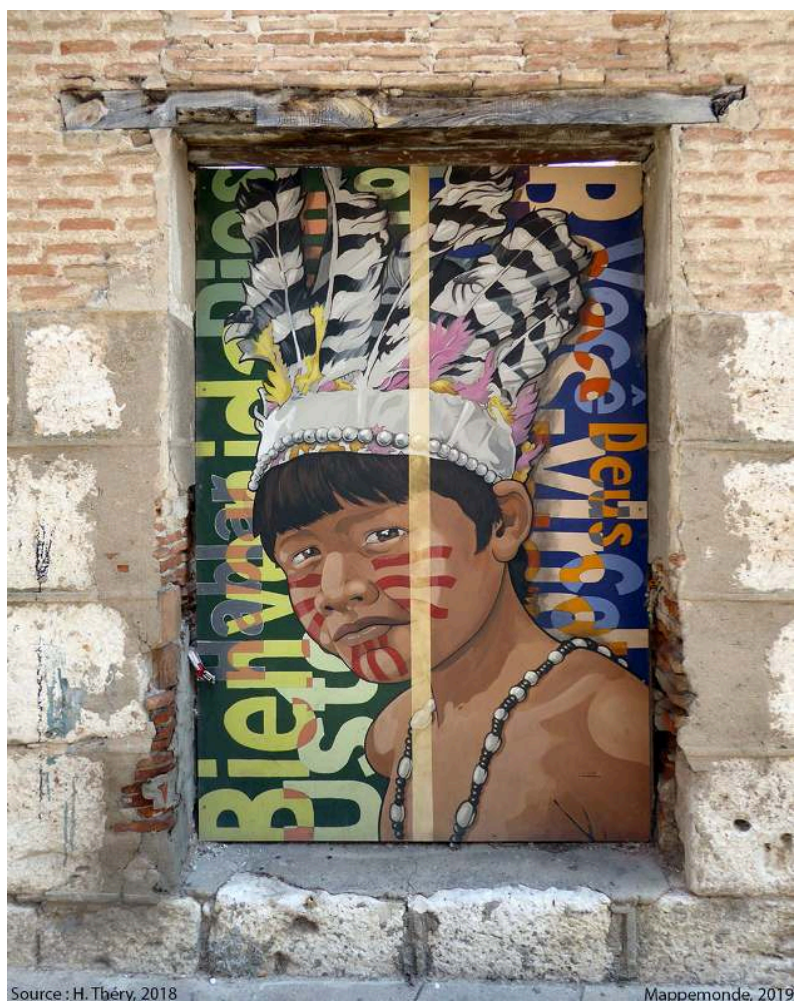

Hervé Théry, 2018

MARIE DESCLAUX (6 juin 2016). « 7 juin 1494. Partage du monde à Tordesillas ». Les Chroniques d'herodote.net. En ligne : https://www.herodote.net/7_juin_1494-evenement-14940607.php

\section{BIBLIOGRAPHIE}

LUÍS ADÃO DA FONSECA ET MARIA CRISTINA CUNHA, dir. (1991). O Tratado de Tordesilhas e a diplomacia lusocastelhana no século XV., Lisbonne : Éd. Inapa, 91 p. ISBN : 972-9019-36-3

RÉGIS DEBRAY (1991). Christophe Colomb, le visiteur de l'aube. Suivi des Traités de Tordesillas (traduits de l'espagnol par Bernard Lesfargues et présentés par Bartolomé Bennassar). Paris : La Différence, 124 p. ISBN : 2-7291-0727-4

MARGARITA GOKUN (2017). ‘Como é Tordesilhas, a cidade onde espanhóis e portugueses dividiram o mundo entre si'. SilverBBC Travel. En ligne : https://www.bbc.com/portuguese/verttra-40655672 


\section{Webographie}

Ayuntamiento de Tordesillas: http://www.tordesillas.net/fr/descubre-tordesillas/historia/eltratado-de-tordesillas

Traité de Tordesillas : https://fr.wikipedia.org/wiki/Trait\%C3\%A9_de_Tordesillas

\section{NOTES}

1. Alors que les autres grands traités ont été signés dans de grandes villes, comme Vienne (1815), Munich (1938) ou Bruxelles (1948), des lieux prestigieux, symboliques ou offrant des commodités aux négociateurs du fait de leurs équipements hôteliers.

2. Certains historiens affirment qu'en réalité les Portugais avaient déjà connaissance de la présence du (futur) Brésil et ont fait en sorte à Tordesillas qu'il soit inclus dans leur partie du Nouveau Monde.

3. Alors encore nommé «Cap des Tempêtes» et rebaptisé après son franchissement, qui permettait d'atteindre les Indes en profitant des vents de mousson.

4. Après quoi les Portugais n'eurent plus qu'à « repousser le méridien » (de Tordesillas), ce qu'ils firent de près de 3000 kilomètres. Principalement en profitant de la période où le roi d'Espagne portait aussi la couronne portugaise (1580-1640), ce qui leur permit de lui laisser croire que ces empiètements ne tiraient pas à conséquence...

5. Où eurent lieu les deux tentatives d'implantation française, la «France Antarctique » du Nicolas Durand de Villegaignon, dans la baie de la (future) Rio de Janeiro (1555) et la «France équinoxiale " Daniel de La Touche, seigneur de la Ravardière, dans le Maranhão (1612), cf. Laurent Vidal, «La présence française dans le Brésil colonial au XVI ${ }^{\mathrm{e}}$ siècle », Cahiers des Amériques latines 34, 2000, URL : http://journals.openedition.org/cal/6486 ; DOI : 10.4000/cal.6486 6. Où elles durent se contenter des trois Guyanes, française, hollandaise et anglaise, dans un angle mort entre les domaines espagnol et portugais.

7. Que seraient la gastronomie et même l'alimentation européenne sans les plantes américaines? L'Italie sans le maïs de la polenta et la tomate de la pizza? L'Irlande et la Bretagne sans pomme de terre ? La Belgique et la Suisse sans le chocolat?

8. Population estimée de 8130 habitants en 2015.

9. La même année que la campagne - et la retraite - de Russie, encore plus désastreuse.

\section{AUTEUR}

\section{HERVÉ THÉRY}

Directeur de recherche émérite au CNRS. Professor visitante na Universidade de São Paulo (USP) Co-directeur de la revue Confins (https://journals.openedition.org/confins) Blog de recherche Braises (https://braises.hypotheses.org) 